\title{
RANDOM TRANSCEIVER NETWORKS
}

\author{
PAUL BALISTER * AND \\ BÉLA BOLLOBÁS, ** University of Memphis \\ MARK WALTERS, ${ }^{* * *}$ University of Cambridge
}

\begin{abstract}
Consider randomly scattered radio transceivers in $\mathbb{R}^{d}$, each of which can transmit signals to all transceivers in a given randomly chosen region about itself. If a signal is retransmitted by every transceiver that receives it, under what circumstances will a signal propagate to a large distance from its starting point? Put more formally, place points $\left\{x_{i}\right\}$ in $\mathbb{R}^{d}$ according to a Poisson process with intensity 1 . Then, independently for each $x_{i}$, choose a bounded region $A_{x_{i}}$ from some fixed distribution and let $g$ be the random directed graph with vertex set $\left\{x_{i}\right\}$ and edges $\overrightarrow{x_{i}} x_{j}$ whenever $x_{j} \in x_{i}+A_{x_{i}}$. We show that, for any $\eta>0, g$ will almost surely have an infinite directed path, provided the expected number of transceivers that can receive a signal directly from $x_{i}$ is at least $1+\eta$, and the regions $x_{i}+A_{x_{i}}$ do not overlap too much (in a sense that we shall make precise). One example where these conditions hold, and so gives rise to percolation, is in $\mathbb{R}^{d}$, with each $A_{x_{i}}$ a ball of volume $1+\eta$ centred at $x_{i}$, where $\eta \rightarrow 0$ as $d \rightarrow \infty$. Another example is in two dimensions, where the $A_{x_{i}}$ are sectors of angle $\varepsilon \theta$ and area $1+\eta$, uniformly randomly oriented within a fixed angle $(1+\varepsilon) \theta$. In this case we can let $\eta \rightarrow 0$ as $\varepsilon \rightarrow 0$ and still obtain percolation. The result is already known for the annulus, i.e. that the critical area tends to 1 as the ratio of the radii tends to 1 , while it is known to be false for the square $\left(l_{\infty}\right)$ annulus. Our results show that it does however hold for the randomly oriented square annulus.
\end{abstract}

Keywords: Random network, random geometric graph, percolation

2000 Mathematics Subject Classification: Primary 60K35

Secondary 82B 43

\section{Introduction}

We consider an ad-hoc wireless network consisting of many transceivers randomly distributed over a large region, some or all of which have sensors that record some local data. Each transceiver can transmit its data to some nearby region. All the recipients of this information then retransmit it. Under some conditions (e.g. enough power and enough transceivers), the information can propagate a large distance. In particular, if we listen at the boundary of the large region, we may still pick up information originating from sensors in the

Received 11 November 2004; revision received 22 February 2009.

* Postal address: University of Memphis, Department of Mathematics, Dunn Hall, 3725 Noriswood, Memphis, TN 38152, USA.

Research supported by NSF grant EIA-0130352.

** Current address: Department of Pure Mathematics and Mathematical Statistics, University of Cambridge, Cambridge CB3 0WB, UK.

Research supported in part by NSF grants DMS-0505550, CNS-0721983, CCF-0728928, and EIA-0130352, and an ARO grant W911NF-06-1-0076.

*** Current address: School of Mathematical Sciences, Queen Mary, University of London, Mile End Road, London E1 4NS, UK. Email address: m.walters@qmul.ac.uk 
centre of the region (see [6]). We wish to prove some bounds on the power and number of transceivers needed for this to occur.

A very natural way to model this is to suppose that the transceivers are distributed according to a Poisson process in some region, for example a square, and that the transceivers are omni-directional, i.e. that any transceiver within a disc about the transceiver can receive the information (where the radius of the disc may depend on the power of the transceiver). A limiting version of this problem is to suppose that the transceivers are distributed according to a Poisson process in the entire plane and ask for the existence of an infinite component; i.e. whether percolation occurs (see [5] and [8]). In this case it is known that we need the power to be such that, on average, a transceiver can broadcast to approximately 4.512 other transceivers (see [10] for numerical simulations and [4] for a semi-rigorous result). It is natural to ask whether we can do better if we transmit to some other region, in particular whether directional transmission can help. In this paper we show that, given any reasonable model of directional transceivers, we can do better. Moreover, this can be achieved with the transceivers randomly oriented; in particular, the transceivers do not require any global knowledge of the arrangement.

To model this setup, fix a probability distribution $\mathcal{D}$ on measurable regions $A \subseteq \mathbb{R}^{d} \backslash\{0\}$. Construct a random digraph $g$ by placing points $\left\{x_{i}\right\}$ in $\mathbb{R}^{d}$ according to a Poisson process with intensity 1 . Choose independently for each $x_{i}$, regions $A_{x_{i}}$ according to the distribution $\mathcal{D}$. Let the vertices of $g$ be the $x_{i}$, and let the edges $x_{i} x_{j}$ lie in $g$ when $x_{j} \in A\left(x_{i}\right):=x_{i}+A_{x_{i}}$. The points $x_{i}$ represent the locations of our transceivers, and the sets $A\left(x_{i}\right)$ represent the region in which the signal strength of $x_{i}$ is sufficiently strong to be received by another transceiver. We assume that the reception of a signal is not directional, so all transceivers within $A\left(x_{i}\right)$ can receive data from $x_{i}$. Individual transceivers may be strongly directional, so $A\left(x_{i}\right)$ may be highly nonsymmetric. Indeed, even the distribution $\mathscr{D}$ may be very irregular, due to variation in power, or partial failure of the transceivers, or even global bias in the direction of transmission. We wish to know under what circumstances $g$ has an infinite directed path.

We shall show that for an infinite directed path to exist we need only the expected volume of $A_{x_{i}}$ to be slightly more than 1, provided there is not much overlap in the regions $A_{x_{i}}$ (in a sense that will be made precise below), and the distribution $\mathscr{D}$ satisfies some mild boundedness conditions.

Throughout this paper, we shall denote by $|S|$ the standard Lebesgue measure of $S$ in $\mathbb{R}^{d}$. We shall fix a distribution $\mathscr{D}$ on regions, and define two distributions derived from $\mathscr{D}$. Let $\mathscr{D}_{0}$ be the distribution of the number of points, $N$, of our Poisson process in $A$, where $A$ is distributed according to $\mathscr{D}$. Note that if all the volumes $|A|$ are the same then $\mathscr{D}_{0}$ is a Poisson distribution with mean $|A|$. Let $\mathcal{D}_{c}$ be the probability distribution on $\mathbb{R}^{d}$ given by

$$
\mathrm{P}_{D_{c}}(S)=\frac{\mathrm{E}(|S \cap A|)}{\mathrm{E}(|A|)},
$$

where $A$ is distributed according to $\mathscr{D}$. In other words, $\mathscr{D}_{c}$ is the unconditioned probability distribution of the location of a 'typical' neighbour of the point 0 .

We now introduce several parameters that describe $\mathscr{D}$. The most important of these, and the most difficult to describe, is the parameter $\delta$ which will indicate the amount of overlap we obtain between the regions $A\left(x_{i}\right)$.

We require, for any $x_{1}$ and $x_{2}$ and fixed $A\left(x_{1}\right)$ and $A\left(x_{2}\right)$, that the probability of a randomly chosen neighbour of a randomly chosen neighbour of $x_{1}$ being a neighbour of $x_{2}$ is at most $\delta$. In addition, we also need the same result if we, rather artificially, replace $A_{x_{1}}$ by $-A_{x_{1}}$. More 
formally, let the sets $A$ and $A^{\prime}$ be distributed according to $\mathcal{D}$. Fixing $A, A^{\prime}$, and $z \in \mathbb{R}^{d}$, let $X$ be a random point uniformly distributed in $A$, and let $Y$ be an independent random variable with distribution $\mathscr{D}_{c}$. Then define $\delta$ by

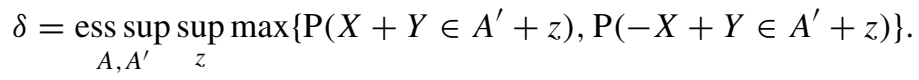

(If $A\left(x_{1}\right)=A+x_{1}, A\left(x_{2}\right)=A^{\prime}+x_{2}$, and $z=x_{2}-x_{1}$, then we obtain the previous description.) Note that if $\mathscr{D}$ is centrally symmetric then the $\mathrm{P}\left(-X+Y \in A^{\prime}+z\right)$ term above is unnecessary since we can replace $A$ by $-A$. Note also that by changing $\mathscr{D}$ on a set of measure 0 we may assume that 'ess sup' is the same as 'sup'.

To illustrate this definition, consider the distribution $\mathscr{D}$ which gives a fixed region $A$ with probability 1 . Then $\delta$ is given more simply by

$$
\delta=|A|^{-2} \sup _{z} \int_{A-z}|(A+x) \cap A| \mathrm{d} x .
$$

(Write $x=X \mp z$ and $A^{\prime}=A$, and note that $X$ and $Y$ are now both uniformly distributed in $A$.) For example, suppose that $A$ is a $d$-dimensional sphere with radius $r$ centred at the origin in $\mathbb{R}^{d}$. Then $(A+x) \cap A$ is contained in a sphere of radius $r \sqrt{1-\alpha^{2} / 4}$ about the point $x / 2$, where $\alpha=\|x\|$. Hence, $|(A+x) \cap A| \leq\left(1-\alpha^{2} / 4\right)^{d / 2}|A|$. Since $|(A+x) \cap A|$ is decreasing for increasing $\|x\|$, the supremum in (2) occurs when $z=0$. Thus,

$$
\delta \leq \int_{0}^{1}\left(1-\frac{\alpha^{2}}{4}\right)^{d / 2}\left(d \alpha^{d-1}\right) \mathrm{d} \alpha .
$$

Substituting $\alpha=\mathrm{e}^{-z}$ and using $1-\alpha^{2} / 4=1-\frac{1}{4} \mathrm{e}^{-2 z} \leq \frac{3}{4}+\frac{1}{2} z \leq \frac{3}{4} \mathrm{e}^{2 z / 3}$ gives

$$
\delta \leq \int_{0}^{\infty}\left(\frac{3}{4} \mathrm{e}^{2 z / 3}\right)^{d / 2} d \mathrm{e}^{-z(d-1)} \mathrm{e}^{-z} \mathrm{~d} z=\left(\frac{3}{4}\right)^{d / 2} \int_{0}^{\infty} d \mathrm{e}^{-(2 d / 3) z} \mathrm{~d} z=\frac{3}{2}\left(\frac{3}{4}\right)^{d / 2},
$$

so $\delta$ decreases exponentially with $d$. (By calculating (2) exactly one can show that $\delta=$ $\frac{3}{2}\left(\int_{0}^{\pi / 3} \sin ^{d} \theta \mathrm{d} \theta\right) /\left(\int_{0}^{\pi / 2} \sin ^{d} \theta \mathrm{d} \theta\right)$. Using this, we can see that the exponential rate of decay given by (3) is sharp, although, for example, $\delta=1-(\sqrt{27} / 4 \pi) \approx 0.5865$ when $d=2$, while (3) gives an estimate larger than 1.)

Another example is in two dimensions with $A$ a randomly oriented thin sector of a disc. This example models the case when the transceivers are highly directional, but not all oriented in the same direction. To be precise, assume that each sector has angle $\varepsilon \theta$, and that the sectors are oriented uniformly over some fixed angle $\theta, 0<\theta \leq 2 \pi$, so that all the sectors lie within an angle of $(1+\varepsilon) \theta$ (or $2 \pi$ if $(1+\varepsilon) \theta \geq 2 \pi$ ). Then the probability distribution $\mathscr{D}_{c}$ has density at most $\varepsilon /|A|$ at any point. It is then clear that $\pm X+Y$ has probability density at most $\varepsilon /|A|$ anywhere, so $\delta=\sup _{z, A, A^{\prime}} \mathrm{P}\left( \pm X+Y \in A^{\prime}+z\right) \leq(\varepsilon /|A|)\left|A^{\prime}\right|=\varepsilon$. More generally, we have the following result.

Lemma 1. If almost all volumes $|A|$ given by $\mathcal{D}$ are the same and, for almost all fixed $z \in \mathbb{R}^{d}$, the probability that $z \in A$ ( $A$ distributed according to $\mathscr{D})$ is at most $\varepsilon$, then $\delta \leq \varepsilon$.

Note that in our example, for $\delta$ to be small, it is necessary that $\varepsilon$ be small. It is not sufficient that $\theta$ be small. Indeed, reducing $\theta$ has little effect, since by applying a suitable area-preserving linear transformation, a small $\theta$ is the same as taking large $\theta$ and replacing the thin sectors by 
appropriate triangular-shaped regions. It is then not hard to see that if $\varepsilon$ fails to be small, $\delta$ will also not be small. Using Theorem 2, we can also show that in this case the area of the sectors $A$ must be significantly larger than 1 if an infinite directed path is to exist.

We should also note that in Lemma 1 , the condition that $|A|$ is constant is required. For example, consider the case when $A=\varnothing$ with probability $1-\varepsilon$ and $A$ is a disc of area $C / \varepsilon$ with probability $\varepsilon$. The probability that $z \in A$ is always at most $\varepsilon$; however, $\delta$ is the same as if $A$ were a disc of area $C$ with probability 1 , and is therefore independent of $\varepsilon$. Indeed, the behaviour of these two models is essentially the same since we can remove the points of the Poisson process in the first model where $A_{x_{i}}=\varnothing$ without altering the percolation properties. But then we obtain the second model scaled up by a factor $1 / \varepsilon$ in area. Moreover, the second model percolates only for $C$ larger than some critical value which is known to be at least 2 (see [9]), and experimental evidence suggests that this value is about 4.512 (see [10]); hence, in both models we need significantly more than one neighbour on average to ensure infinite directed paths.

The other parameters we need are somewhat easier to describe. Define $\eta$ so that $1+\eta$ is the average number of neighbours of a point in $g$, which is also the average volume $\mathrm{E}(|A|)$. Define $\sigma^{2}$ to be the variance of the number of neighbours of a point. We shall see below that $\sigma^{2}=\mathrm{E}(|A|)+\operatorname{var}(|A|)$, so $\sigma^{2} \geq 1+\eta$ with equality if and only if the volume $|A|$ is almost surely constant. We shall assume that all sets $A$ given by our distribution $\mathscr{D}$ lie in a ball of radius $r_{0}$ about 0 . We also assume that the root mean-square distance of a neighbour is at least $r_{m}>0$ in any direction, i.e.

$$
r_{m}^{2} \leq \mathrm{E}\left((Y \cdot u)^{2}\right) \quad \text { for any unit vector } u,
$$

where $Y$ is distributed according to $\mathscr{D}_{c}$. We can now state the main result.

Theorem 1. There is an absolute constant $c>0$ such that if $\eta \leq 1$ and

$$
\delta<c r_{m}^{9} r_{0}^{-9} \eta^{16} \sigma^{-32}
$$

then $g$ almost surely has an infinite directed path.

From Theorem 1 we can deduce two immediate corollaries.

Corollary 1. Assume that $\mathrm{g}$ consists of the points of a Poisson process with intensity 1 in $\mathbb{R}^{d}, d \geq 2$, and that each point is joined to all other points within a ball of volume $1+\eta$. Then there exists a constant $c>0$, independent of $d$, such that if $\eta>c(0.9911)^{d}$ then $g$ almost surely has an infinite component.

Proof. The distribution $\mathcal{D}$ gives the ball with volume $1+\eta$ with probability 1 . An infinite directed path is an infinite component since $\overrightarrow{x y} \in \mathcal{g}$ if and only if $\overrightarrow{y x} \in \mathcal{g}$. From the above discussion, $\delta \leq 1.5(0.75)^{d / 2}$. But $r_{0} / r_{m}$ is bounded by a polynomial in $d$ (in fact, $r_{0} / r_{m}=\sqrt{d+2}$ ) and $\sigma^{2}=1+\eta$ is bounded when $\eta \leq 1$. The result follows for large $d$ since $0.9911^{16}>\sqrt{0.75}$. Note that, for any $d \geq 2$, there does exist some $\eta$ such that $g$ has an infinite component. To see this, divide $\mathbb{R}^{d}$ into cubes of side length $L$ and identify each cube with a point in $\mathbb{Z}^{d}$ in the obvious manner. Define a site percolation on $\mathbb{Z}^{d}$ by declaring a site open if the corresponding cube contains a point of the Poisson process. Assume that $\eta$ is such that the ball of volume $1+\eta$ has radius at least $L \sqrt{(d-1)+4}$. Then any pair of points in adjacent cubes are joined. Thus, if the site percolation on $\mathbb{Z}^{d}$ has an infinite open component then $g$ must also have an infinite component. Thus, provided that we choose $L$ such that $1-\exp \left(-L^{d}\right)$ is 
more than the critical probability of site percolation in $\mathbb{Z}^{d}$ (which is known to be strictly less than 1), then $g$ almost surely has an infinite component. Hence, by increasing $c$ if necessary, the result holds for all $d \geq 2$.

Corollary 2. Assume that $\mathrm{g}$ consists of the points of a Poisson process with intensity 1 in $\mathbb{R}^{2}$, and that each point is joined to all other points within a sector of a disc of area $1+\eta$ and angle $\varepsilon \theta$, randomly oriented over a fixed angle of $\theta$. Then there exists a constant $c>0$, independent of both $\theta$ and $\varepsilon$, such that if $\eta>c \varepsilon^{1 / 16}$ then $g$ almost surely has an infinite directed path.

Proof. From the above discussion we have $\delta \leq \varepsilon$. Assume first that $\varepsilon$ is bounded and that $\eta \leq 1$, so $\sigma^{2} \leq 2$. For $\theta$ bounded away from $0, r_{0} / r_{m}$ is bounded and the result follows from Theorem 1 for some $c$. As $\theta \rightarrow 0, r_{0} / r_{m} \rightarrow \infty$, but if we apply an area-preserving linear transformation of $\mathbb{R}^{2}$, we can make all the sensor regions approximately triangular with $r_{0} / r_{m}$ bounded. Thus, we can choose $c$ independently of $\theta$ for all $\theta>0$. Since increasing the radius of the sectors only makes percolation more likely, the result follows for all $\eta$ when $\varepsilon$ is sufficiently small $\left(c \varepsilon^{1 / 16}<1\right)$.

For larger $\varepsilon$, note that if $g$ has an infinite directed path for $\varepsilon=\varepsilon_{0}$ with $\eta=1$ then $g$ still has an infinite directed path if we increase $\varepsilon$ (and, hence, $\eta$ ) while keeping the radius of the sectors the same. Hence, by increasing $c$ if necessary, the result follows for all $\varepsilon \in(0,2]$. For $\varepsilon>2$, the sectors always contain a fixed sector of angle $(\varepsilon-1) \theta$, and, thus, a triangle of area some constant fraction of $1+\eta$. By a suitable linear transformation we can make these triangles equilateral, so percolation will occur once this area is above some absolute constant, independently of the shape of the original triangle. Thus, by increasing $c$ again if necessary, we obtain the result for all $\varepsilon$.

For another model, take $A$ to be a fixed annulus of area $1+\eta$ and inner and outer radii $r(1-\varepsilon)$ and $r$, respectively, where $r=r(\eta, \varepsilon)$ is determined by the requirement that $|A|=\pi r^{2}(2-$ $\varepsilon) \varepsilon=1+\eta$. In this case, transceivers transmit data to other transceivers only if they are placed a distance between $r(1-\varepsilon)$ and $r$ apart. For this model, it is already known that, for any $\eta>0$, we obtain percolation for sufficiently small $\varepsilon$ (see [3] and [7]). In [3] it was also shown that this result fails for a 'square' annulus, defined by $A=\{(x, y): r(1-\varepsilon) \leq \max \{|x|,|y|\} \leq r\}$. Indeed, we require that $\eta>0.014$ for all $\varepsilon>0$. For the square annulus, it can be shown that $\delta \geq \frac{9}{64}$ for any $\varepsilon>0$, unlike the case of the usual annulus where $\delta \rightarrow 0$ as $\varepsilon \rightarrow 0$. On the other hand, the randomly oriented square annulus does have small $\delta$ by Lemma 1 , so in this case we do obtain percolation for any fixed $\eta>0$ if $\varepsilon$ is sufficiently small.

\section{Lower bound}

Before we give the proof of Theorem 1, we first note that we can give a positive lower bound on $\eta$ that is necessary for an infinite directed path to exist in $g$. Recall that all regions $A$ are assumed to lie within a ball of radius $r_{0}$ about 0 and that the average number of neighbours is $1+\eta$.

Theorem 2. Suppose that $\eta<(2 K)^{-K} / 3$, where $K=\left\lceil\left(4 r_{0}\right)^{d}+1\right\rceil$. Then there is almost surely no infinite directed path in $\mathrm{g}$.

Proof. Fix $x_{0}$ in the process. Let $N_{i}$ be the number of points at graph distance $i$ from $x_{0}$ in $g$. We need to show that, almost surely, $N_{i}=0$ for some $i$. For all $i, \mathrm{E}\left(N_{i+1} \mid N_{i}\right) \leq(1+\eta) N_{i}$, since this is true even conditioning on the choice of $A(x)$ and the Poisson process in $A(x)$ for all $x$ within a graph distance $i-1$ of $x_{0}$. (All the points at graph distance $i+1$ lie outside 
these regions and are contained within a union of $N_{i}$ regions, each of expected volume $1+\eta$.) Thus, $\mathrm{E}\left(N_{i+1}\right)=\mathrm{E}\left(\mathrm{E}\left(N_{i+1} \mid N_{i}\right)\right) \leq(1+\eta) \mathrm{E}\left(N_{i}\right)$. In particular, when $\eta<0, \mathrm{E}\left(N_{i}\right) \rightarrow 0$ as $i \rightarrow \infty$, and so almost surely some $N_{i}=0$. Hence, we may assume that $\eta \geq 0$. Now $\mathrm{E}\left(N_{2} \mid N_{1}\right) \leq(1+\eta) N_{1}$. All the $N_{2}$ points at graph distance 2 from $x_{0}$ lie within $2 r_{0}$ of $x_{0}$ and outside $A\left(x_{0}\right)$. Thus, we also have $\mathrm{E}\left(N_{2} \mid N_{1}\right) \leq\left(4 r_{0}\right)^{d}$, since this is true even conditioning on $A\left(x_{0}\right)$ and the Poisson process inside $A\left(x_{0}\right)$. Combining these results we have

$$
\begin{aligned}
\mathrm{E}\left(N_{2}\right) & =\mathrm{E}\left(\mathrm{E}\left(N_{2} \mid N_{1}\right)\right) \\
& \leq \mathrm{E}\left(\min \left\{(1+\eta) N_{1},\left(4 r_{0}\right)^{d}\right\}\right) \\
& \leq(1+\eta) \mathrm{E}\left(N_{1}\right)-1 \mathrm{P}\left(N_{1} \geq\left(4 r_{0}\right)^{d}+1\right) \\
& \leq(1+\eta)^{2}-\mathrm{P}\left(N_{1} \geq\left(4 r_{0}\right)^{d}+1\right) .
\end{aligned}
$$

Let $A=A\left(x_{0}\right)$ be the neighbourhood for $x_{0}$. Then, since $|A|<\left(2 r_{0}\right)^{d}$ and $\mathrm{E}(|A|) \geq 1$,

$$
\mathrm{P}\left(|A|>\frac{1}{2}\right)>\frac{1}{2\left(2 r_{0}\right)^{d}} .
$$

Also, conditional on $|A|>\frac{1}{2}$, the number of points in $A$ stochastically dominates a Poisson distribution with mean $\frac{1}{2}$. Thus,

$$
\mathrm{P}\left(N_{1} \geq\left(4 r_{0}\right)^{d}+1|| A \mid>\frac{1}{2}\right) \geq \frac{\mathrm{e}^{-1 / 2}}{2^{K} K !},
$$

where $K=\left\lceil\left(4 r_{0}\right)^{d}+1\right\rceil$. Therefore,

$$
\mathrm{P}\left(N_{1} \geq\left(4 r_{0}\right)^{d}+1\right) \geq \frac{1}{2\left(2 r_{0}\right)^{d}} \frac{\mathrm{e}^{-1 / 2}}{2^{K} K !}>(2 K)^{-K}>3 \eta>2 \eta+\eta^{2},
$$

by assumption on $\eta$. Hence, $\mathrm{E}\left(N_{2}\right)<(1+\eta)^{2}-\left(2 \eta+\eta^{2}\right)=1$. However, it is clear that $\mathrm{E}\left(N_{i+2} \mid N_{i}\right) \leq \mathrm{E}\left(N_{2}\right) N_{i}$, so $\mathrm{E}\left(N_{2 i}\right) \leq\left(\mathrm{E}\left(N_{2}\right)\right)^{i}$, which tends to 0 as $i \rightarrow \infty$. The result now follows.

\section{Proof of Theorem 1}

First we convert the definition of $\delta$ into a more usable form.

Lemma 2. We can define sets $B\left(x_{i}\right)$ depending on $A\left(x_{i}\right)$ so that if $z \notin B\left(x_{i}\right)$ and $A$ is distributed according to $\mathscr{D}$ then, conditional on any value of $A\left(x_{i}\right)$, we have

$$
\mathrm{E}\left(\left|(z+A) \cap A\left(x_{i}\right)\right|\right) \leq \sqrt{\delta} \mathrm{E}(|A|) .
$$

Moreover, for all $z \in \mathbb{R}^{d}$ and $\mathcal{D}$-almost all choices of $A$ and $A\left(x_{i}\right)$,

$$
\left|(z \pm A) \cap B\left(x_{i}\right)\right| \leq \sqrt{\delta}|A| .
$$

Proof. Fix $A\left(x_{i}\right)$, and define

$$
B\left(x_{i}\right)=\left\{z \in \mathbb{R}^{d}: \mathrm{E}\left(\left|(z+A) \cap A\left(x_{i}\right)\right|\right)>\sqrt{\delta} \mathrm{E}(|A|)\right\} .
$$


The first inequality then holds automatically. Now let $X$ be uniformly distributed in $A$, and let $Y$ be distributed according to the distribution $\mathscr{D}_{c}$. Then, for either sign choice of ' \pm ' (fixed throughout) and almost all choices of $A$ and $A\left(x_{i}\right)$,

$$
\begin{aligned}
\delta & \geq \mathrm{P}\left( \pm X+Y \in A\left(x_{i}\right)+z\right) \\
& \geq \mathrm{P}\left( \pm X+Y \in A\left(x_{i}\right)+z \mid \pm X \in B\left(x_{i}\right)+z\right) \mathrm{P}\left( \pm X \in B\left(x_{i}\right)+z\right) \\
& =\mathrm{P}\left(W+Y \in A\left(x_{i}\right) \mid W \in B\left(x_{i}\right)\right) \mathrm{P}\left( \pm X \in B\left(x_{i}\right)+z\right) \\
& =\frac{\mathrm{E}\left(\left|\left(W+A^{\prime}\right) \cap A\left(x_{i}\right)\right| \mid W \in B\left(x_{i}\right)\right)}{\mathrm{E}\left(\left|A^{\prime}\right|\right)} \frac{\left| \pm A \cap\left(B\left(x_{i}\right)+z\right)\right|}{|A|} \\
& \geq \frac{\sqrt{\delta}\left|((-z) \pm A) \cap B\left(x_{i}\right)\right|}{|A|}
\end{aligned}
$$

where $A^{\prime}$ is distributed according to $\mathscr{D}$ and $W= \pm X-z$. Hence, $\left|(z \pm A) \cap B\left(x_{i}\right)\right| \leq \sqrt{\delta}|A|$ for all $z \in \mathbb{R}^{d}$.

The sets $B\left(x_{i}\right)$ give regions around $x_{i}$ that we want to avoid, since if $x_{j} \in B\left(x_{i}\right)$ then the neighbourhoods of $x_{j}$ and $x_{i}$ may have a large intersection. In this case we will typically have too few 'new' neighbours of $x_{j}$, say, that we have not already encountered when looking for neighbours of $x_{i}$. Ideally, we would like to have $B\left(x_{i}\right)=\varnothing$, but in general all we can guarantee is that $B\left(x_{i}\right)$ has small intersection with every region $A\left(x_{k}\right)$.

We introduce the following parameters of the distribution $\mathscr{D}_{0}$ governing $N$, the number of neighbours of a point:

$$
\mathrm{E}(N)=1+\eta, \quad \operatorname{var}(N)=\sigma^{2}, \quad \mathrm{P}(N=0)=p_{0} .
$$

Since the regions $A$ are bounded, $N$ is stochastically bounded by a Poisson variable with finite mean. Hence, all moments, including $\operatorname{var}(N)$, are finite, and $p_{0}>0$. By Theorem 2, if $\eta \leq 0$ then there will almost surely be no infinite directed path in $g$; hence, we shall always assume that $\eta>0$. We also define $\lambda>0$, so that

$$
\mathrm{E}\left(\mathrm{e}^{-\lambda N}\right)=\mathrm{e}^{-\lambda}
$$

Note that, for large $x, \mathrm{E}\left(\mathrm{e}^{-x N}\right)$ tends to $p_{0}>0$ and, for small $x, \mathrm{E}\left(\mathrm{e}^{-x N}\right)=1-x \mathrm{E}(N)+$ $O\left(x^{2}\right)=1-x(1+\eta)+O\left(x^{2}\right)<\mathrm{e}^{-x}$. Hence, by continuity, $\lambda$ does indeed exist.

Lemma 3. If $A$ is distributed according to $\mathscr{D}$ and $\eta>0$ then

$$
\eta=\mathrm{E}(|A|)-1, \quad \sigma^{2}=\operatorname{var}(|A|)+\mathrm{E}(|A|), \quad p_{0} \geq \mathrm{e}^{-(1+\eta)}, \quad \lambda \geq \frac{2 \eta}{\sigma^{2}+(1+\eta)^{2}} .
$$

Proof. Let $A$ be distributed according to $\mathscr{D}$, so that $N$, conditioned on $A$, is distributed as a Poisson variable with mean $|A|$. For the first equation, $1+\eta=\mathrm{E}(N)=\mathrm{E}(\mathrm{E}(N \mid A))=\mathrm{E}(|A|)$. Also,

$\sigma^{2}+(\mathrm{E}(N))^{2}=\mathrm{E}\left(N^{2}\right)=\mathrm{E}\left(\mathrm{E}\left(N^{2} \mid A\right)\right)=\mathrm{E}\left(|A|+|A|^{2}\right)=\operatorname{var}(|A|)+(\mathrm{E}(|A|))^{2}+\mathrm{E}(|A|)$.

Since $\mathrm{E}(N)=\mathrm{E}(|A|)$, we obtain the second equality. The probability that $N=0$ is $\mathrm{E}(\mathrm{P}(N=$ $0 \mid A))=\mathrm{E}\left(\mathrm{e}^{-|A|}\right) \geq \mathrm{e}^{-(1+\eta)}$ by convexity of $\mathrm{e}^{-x}$. Finally, $N$ is nonnegative and $\mathrm{e}^{-x} \leq$ $1-x+x^{2} / 2$ for $x \geq 0$. Hence, $\mathrm{E}\left(\mathrm{e}^{-\lambda N}\right) \leq 1-\lambda \mathrm{E}(N)+\lambda^{2} \mathrm{E}\left(N^{2}\right) / 2$. If $0<\lambda<2 \eta / \mathrm{E}\left(N^{2}\right)$ then $\mathrm{E}\left(\mathrm{e}^{-\lambda N}\right)<1-\lambda(1+\eta)+\lambda \eta=1-\lambda<\mathrm{e}^{-\lambda}$, contradicting the definition of $\lambda$. Thus, $\lambda \geq 2 \eta / \mathrm{E}\left(N^{2}\right)=2 \eta /\left(\sigma^{2}+(1+\eta)^{2}\right)$. 
Our aim will be to compare the percolation process with an oriented bond percolation on $\mathbb{Z}^{2}$. First we shall use a linear transformation to make $\mathscr{D}$ somewhat more symmetric. If we apply a volume-preserving linear transformation to $\mathbb{R}^{d}$ then the Poisson process will again give us a Poisson process of intensity 1. Expression (1) defining $\delta$ is unchanged under any such transformation, as are the parameters $\eta, \sigma, \lambda$, and $p_{0}$, which depend only on $\mathscr{D}_{0}$. If $Y$ is distributed according to $\mathscr{D}_{c}$ then $\mathrm{E}\left((Y \cdot u)^{2}\right)$ is a positive definite quadratic form in $u$, so by a suitable volume-preserving linear transformation we can ensure that $\mathrm{E}\left((Y \cdot u)^{2}\right)=r_{m}^{\prime 2}\|u\|^{2}$ for all $u$ and some $r_{m}^{\prime}$, the analogue of $r_{m}$ for the transformed process. All the parameters of Theorem 1 remain unaltered under this transformation except for $r_{0}$ and $r_{m}$. Before this transformation, $\mathrm{E}\left((Y \cdot u)^{2}\right) \geq r_{m}^{2}\|u\|^{2}$ and $|Y \cdot u| \leq r_{0}\|u\|$ for all $u$, so

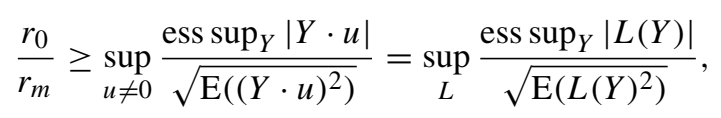

where $L$ runs over all nonzero linear functionals. This last expression however is invariant under any linear transformation and is equal to $r_{0}^{\prime} / r_{m}^{\prime}$, where $r_{0}^{\prime}$ is the $r_{0}$ for the transformed process. Hence, $r_{0}^{\prime} / r_{m}^{\prime} \leq r_{0} / r_{m}$. Since Theorem 1 depends on $r_{m}$ and $r_{0}$ only via this ratio, it is enough to prove the result for this transformed version.

If the distribution $\mathscr{D}_{c}$ has drift, we shall however apply a second volume-preserving linear transformation. We shall define a large constant $C=C(\sigma, \eta)>1$ which is a function of $\sigma$ and $\eta$ only (and, hence, is unaffected by any volume-preserving transformation on $\mathbb{R}^{d}$ ). Now $\operatorname{var}(Y \cdot u)+\left(C r_{0} / r_{m}\right)^{2}(\mathrm{E}(Y \cdot u))^{2}$ is also a positive definite quadratic form in $u$, so we can apply a volume-preserving transformation such that

$$
\operatorname{var}(Y \cdot u)+\left(\frac{C r_{0}}{r_{m}}\right)^{2}(\mathrm{E}(Y \cdot u))^{2}=\mathrm{E}\left((Y \cdot u)^{2}\right)+\left(\left(\frac{C r_{0}}{r_{m}}\right)^{2}-1\right)(\mathrm{E}(Y \cdot u))^{2}=r_{s}^{2}\|u\|^{2}
$$

for some constant $r_{s}>0$. Since $C r_{0} / r_{m}>1$, this transformation compresses vectors in the direction of $\mathrm{E}(Y)$ and expands vectors orthogonal to $\mathrm{E}(Y)$ by a factor $r_{s} / r_{m}$. Hence, at worst, $r_{0}$ increases by a factor $r_{s} / r_{m}$. From now on we shall replace $r_{0}$ by $r_{1}=r_{0} r_{s} / r_{m}$ and $r_{m}$ by $r_{s}$ in the statement of Theorem 1, noting that $r_{1}$ is still a bound on the size of the transformed regions $A_{x_{i}}$. Finally, we shall rotate the process so that the drift $\mathrm{E}(Y)$, if nonzero, points in the direction $(1,1,0,0, \ldots)$ in $\mathbb{R}^{d}$. Define $R=C r_{1}$. Then to summarise, in our transformed process

$$
r_{s}^{2} \operatorname{var}(Y \cdot u)+R^{2}(\mathrm{E}(Y \cdot u))^{2}=r_{s}^{4} \quad \text { and } \quad|Y \cdot u| \leq r_{1}
$$

for any unit vector $u$, and we need to show that $g$ almost surely has an infinite directed path under the assumption that

$$
\delta<c r_{s}^{9} r_{1}^{-9} \eta^{16} \sigma^{-32} .
$$

Partition $\mathbb{R}^{2}$ into $6 R \times 6 R$ squares, and let the site $x \in \mathbb{Z}^{2}$ correspond to the cylinder $C_{x}=$ $\left(6 R x+[-3 R, 3 R)^{2}\right) \times \mathbb{R}^{d-2}$ in $\mathbb{R}^{d}=\mathbb{R}^{2} \times \mathbb{R}^{d-2}$. More generally, write $C_{x, k}, k=1,2,3$, for the cylinder $C_{x, k}=\left(6 R x+[-k R, k R)^{2}\right) \times \mathbb{R}^{d-2}$ (see Figure 1 for the case in which $d=2$ ). Our bonds $x y$ in $\mathbb{Z}^{2}$ will correspond to certain good events in the corresponding cylinder $C_{x} \cup C_{y}$ with two-dimensional $6 R \times 12 R$ rectangular cross sections. Throughout most of what follows, we shall mostly be interested only in the first two coordinates of the points of our process, except when intersecting regions $A\left(x_{i}\right)$, in which case we use all $d \geq 2$ dimensions.

Roughly speaking, these good events will be the ability to get from some given set $P$ of $n$ points near the middle of $C_{x}$ to every point of a set $P^{\prime}$ of size $n$ near the middle of $C_{y}$ by paths 


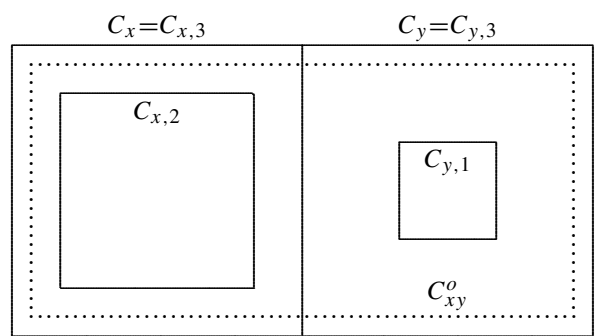

Figure 1: Cylinder sets $C_{x}$ and $C_{y}$.

that lie entirely within $C_{x} \cup C_{y}$. Here $n$ is some fixed large number that will be determined later. Using $n$ points instead of just one increases the chances of success since in practice it is very unlikely that there is a path from a given single point in $C_{x}$ to $C_{y}$. But if there is then there are often many points in $C_{y}$ that we can reach. Nevertheless, provided that we choose our sets consistently (so the $P^{\prime}$ for $x$ becomes the $P$ for $y$ ), an infinite directed path in $\mathbb{Z}^{2}$ from $x$ will result in an infinite path in $g$ from at least one of the points of $P$.

To construct these paths, we shall need to 'explore' the graph $g$. When we encounter a vertex $z$, there are random choices of $A(z)$ and the Poisson process within $A(z)$. If we condition on these, we effectively fix these choices. When we encounter subsequent vertices $w$, the choice of $A(w)$ is independent of this conditioning event, but the Poisson process in $A(w)$ is already determined in $A(z) \cap A(w)$. However, the Poisson process in $A(z) \backslash A(w)$ is independent of the conditioning event. Thus, we can imagine 'growing' $g$ by, at each step, fixing $A(w)$ and the Poisson process in the subset of points of $A(w)$ that do not lie in any previously seen $A(z)$. Since the previous $A(z)$ contain vertices of $g$ that we have encountered already, we want most of $A(w)$ to be new. To do this, we must control the number of regions $A(z)$ we have previously looked at (i.e. conditioned on). Hence, when constructing our paths from $P$ in $C_{x} \cup C_{y}$, we shall allow ourselves to 'test' only regions $A(z)$ around at most $N$ points ( $N$ to be determined below). The set of points we test will be called $Q^{\prime}$. Also, prior to constructing these paths, there will be a set $Q$ of up to $3 N$ points in $C_{x} \cup C_{y}$ that have been tested when constructing earlier bonds, and we shall need to avoid the regions $A(z)$ about these points as well. Since we wish to avoid points in $A(z)$ for $z \notin C_{x} \cup C_{y}$, we shall only consider points at least $r_{1}$ from the boundary of this set, and so we write $C_{x y}^{o}=\left\{z \in C_{x} \cup C_{y}: d\left(z, \partial\left(C_{x} \cup C_{y}\right)\right) \geq r_{1}\right\}$. We shall assume that we can get to $x \in \mathbb{Z}^{2}$ from the origin in our percolation on $\mathbb{Z}^{2}$, and, hence, we can get to every point in $P$. In doing so we have fixed points of $P \cup Q$, the choice of $A(z)$ for $z \in Q$, and the state of the Poisson process in $A(z)$ for $z \in Q$. Note in particular that we have conditioned on an event that implies that there are at least $n$ points in $C_{x}$. Having conditioned on this data, we still have a Poisson process in the remaining region of $C_{x y}^{o}$ and a choice of $A(z)$ for points in this process as well as for $z \in P$. Points outside $C_{x y}^{o}$ will be ignored when considering the bond $x y$ of $\mathbb{Z}^{2}$.

Now we make things more formal. Fix points $P=\left\{x_{1}, \ldots, x_{n}\right\}$ and $Q=\left\{y_{1}, \ldots, y_{k}\right\}, k \leq$ $3 N$, in $C_{x} \cup C_{y}$ corresponding to an bond $x y$ of $\mathbb{Z}^{2}$, where $y=x+(0,1)$ or $y=x+(1,0)$. Fix the choice of neighbourhood regions $A\left(y_{j}\right), y_{i} \in Q$, and the Poisson process within these $A\left(y_{j}\right)$. In what follows, everything will be conditioned on this data. Some regions outside $C_{x y}^{o}$ will also be conditioned on, owing to the construction of previous bonds. Since we shall not consider points outside $C_{x y}^{o}$ when considering the bond $x y$, it will be convenient to assume that we have conditioned on the entire process outside $C_{x y}^{o}$ as well. We shall not 
however condition on the choice of regions $A\left(x_{i}\right), x_{i} \in P$, or on the Poisson process in the rest of $C_{x y}^{o}$. We shall make the following assumptions.

(A1) The points $x_{i}$ lie in the central cylinder $C_{x, 2}$ for all $x_{i} \in P$.

(A2) $x_{i} \notin B\left(y_{j}\right)$ for all $x_{i} \in P$ and all $y_{j} \in Q$.

(A3) $\mathrm{P}\left(x_{i} \in B\left(x_{j}\right)\right) \leq \sqrt[4]{\delta}$ for all $x_{i}, x_{j} \in P, i \neq j$.

In (A3), the choice of $A_{x_{j}}$ and, hence, $B\left(x_{j}\right)$ is random, and the probability refers to this choice which is made in accordance with the distribution $\mathscr{D}$. Assumptions (A2) and (A3) will be required to ensure that the neighbourhoods $A(z)$ that we construct are unlikely to overlap too much. Assumption (A1) avoids problems that may occur near the boundary of $C_{x y}^{o}$.

Given the situation described above, we shall construct sets of points $P^{\prime}=\left\{x_{1}^{\prime}, \ldots, x_{r}^{\prime}\right\}$ and $Q^{\prime}=\left\{y_{1}^{\prime}, \ldots, y_{k^{\prime}}^{\prime}\right\}, r+k^{\prime} \leq N$, of the Poisson process in $C_{x y}^{o}$ with the following properties.

(C1) The points $x_{i}^{\prime}$ lie in the central cylinder $C_{y, 2}$ for all $x_{i}^{\prime} \in P^{\prime}$.

(C2) $x_{i}^{\prime} \notin B(z)$ for all $x_{i}^{\prime} \in P^{\prime}$ and all $z \in P \cup Q \cup Q^{\prime}$.

(C3) $\mathrm{P}\left(x_{i}^{\prime} \in B\left(x_{j}^{\prime}\right)\right) \leq \sqrt[4]{\delta}$ for all $x_{i}^{\prime}, x_{j}^{\prime} \in P^{\prime}, i \neq j$.

(C4) $z \notin A\left(y_{i}\right)$ for all $z \in P^{\prime} \cup Q^{\prime}$ and all $y_{i} \in Q$.

(C5) For all $x_{i}^{\prime} \in P^{\prime}$, some $x_{j} \in P$ are joined to $x_{i}^{\prime}$ by a sequence of points $y_{i_{1}}^{\prime}, \ldots, y_{i_{t}}^{\prime}$ of $Q^{\prime}$.

The construction will depend on the choice of $A(z)$ and the Poisson process restricted to $A(z)$ for $z \in P \cup Q^{\prime}$, but it will not depend on the values of $A(z)$ for $z \in P^{\prime}$. Hence, the probability in (C3), which is over all choices of $A_{x_{j}^{\prime}}$ according to the distribution $\mathscr{D}$, makes sense.

We shall declare the bond $\overrightarrow{x y}$ open with respect to $P$ and $Q$ if in this construction we can take $r=\left|P^{\prime}\right|=n$. In this case, conditions (C1)-(C3) ensure that $P^{\prime}$ and $Q^{\prime}$ can be used in the construction of the next bond $\overrightarrow{y z}$ of $\mathbb{Z}^{2}$. (The $Q$ for $\overrightarrow{y z}$ will include any points of $P \cup Q \cup Q^{\prime}$ that lie in $C_{y z}$.) Note that the openness of $\overrightarrow{x y}$ depends on the choices of $P$ and $Q$ as well as the restriction of the process to the region $C_{x y}^{o}$. Condition (C4) ensures that we never look at the process in $\bigcup_{y_{i} \in Q} A\left(y_{i}\right)$, as these regions will have been tested earlier. Condition (C5) ensures that every point of $P^{\prime}$ is reachable from some point of $P$ in $g$, so that an infinite path in the $\mathbb{Z}^{2}$ process will ensure an infinite path in $g$.

The sets $P$ and $Q$ will depend on the construction of previous bonds, which will introduce complex dependencies between the bonds. Nevertheless, we start by showing that an individual bond is open with high probability, regardless of the choice of $P$ and $Q$ and regardless of the Poisson process in $\bigcup_{y_{i} \in Q} A\left(y_{i}\right)$ or outside $C_{x y}^{o}$.

The proof of the bound is complicated by the fact that the regions $A(z)$ intersect, so we shall first consider the simpler case when we ignore these intersections and model the percolation by a branching process (see, for example, [1, pp. 1-8]). We shall generally refer to the points of a branching process as nodes to avoid confusion with the points of our Poisson process. We shall first prove two simple results about branching processes. The parameters $\eta, \sigma, p_{0}$, and $\lambda$ are as defined before Lemma 3.

Lemma 4. Consider a branching process where at each step each node branches into several new nodes independently according to the distribution $\mathfrak{D}_{0}$. Let $N_{t}$ be the number of nodes at time $t>0$. Then $\mathrm{P}\left(N_{t} \geq(1+\eta)^{t}\right) \geq \eta p_{0}^{2} / \sigma^{2}$. 
Proof. It is easy to show by induction on $t$ (see, e.g. [1, p. 4]) that

$$
\mathrm{E}\left(N_{t}\right)=(1+\eta)^{t} \quad \text { and } \quad \operatorname{var}\left(N_{t}\right)=\frac{\sigma^{2}(1+\eta)^{t-1}\left((1+\eta)^{t}-1\right)}{\eta} .
$$

Let $X_{t}=N_{t} / \mathrm{E}\left(N_{t}\right)$, so that $\mathrm{E}\left(X_{t}\right)=1$ and $\operatorname{var}\left(X_{t}\right)<\sigma^{2} / \eta$. By the Cauchy-Schwarz inequality,

$$
\mathrm{E}\left(\mathbf{1}_{X_{t} \geq 1}\right) \mathrm{E}\left(\left(X_{t}-1\right)^{2}\right) \geq\left(\mathrm{E}\left(\left(X_{t}-1\right) \mathbf{1}_{X_{t} \geq 1}\right)\right)^{2} .
$$

Now

$$
\begin{gathered}
\mathrm{E}\left(\left(X_{t}-1\right)^{2}\right)=\operatorname{var}\left(X_{t}\right)<\frac{\sigma^{2}}{\eta}, \\
\mathrm{E}\left(\left(X_{t}-1\right) \mathbf{1}_{X_{t} \geq 1}\right)=\mathrm{E}\left(\left(1-X_{t}\right) \mathbf{1}_{X_{t} \leq 1}\right) \geq \mathrm{P}\left(X_{t}=0\right) \geq \mathrm{P}\left(N_{1}=0\right)=p_{0} .
\end{gathered}
$$

Hence, $\mathrm{P}\left(N_{t} \geq(1+\eta)^{t}\right)=\mathrm{E}\left(\mathbf{1}_{X_{t} \geq 1}\right) \geq \eta p_{0}^{2} / \sigma^{2}$.

Lemma 5. Consider a branching process where at each step each node branches into several new nodes independently according to the distribution $\mathscr{D}_{0}$. Suppose also that we randomly remove nodes, if necessary, so there are at most $K$ nodes at each step, and assume that $T \leq$ $\lambda \mathrm{e}^{\lambda(K-1)} / 2$. Then the probability that there is at least one node at time $T$ is at least $1-\mathrm{e}^{-\lambda / 2}$.

Proof. Let $N_{t}$ be the number of nodes at time $t$, and consider the random variable $X_{t}$ defined by $X_{t}=\exp \left(-\lambda N_{t}\right)$. Now

$$
N_{t+1}=\min \left(N_{t+1}^{\prime}, K\right), \quad \text { where } \quad N_{t+1}^{\prime}=\sum_{i=1}^{N_{t}} Y_{i}
$$

and $Y_{i}$ are independent random variables with distribution $\mathscr{D}_{0}$. Recall that $\lambda$ is defined so that

$$
\mathrm{E}\left(\exp \left(-\lambda Y_{i}\right)\right)=\mathrm{e}^{-\lambda}
$$

Hence,

$$
\mathrm{E}\left(\exp \left(-\lambda N_{t+1}^{\prime}\right) \mid N_{t}\right)=\prod_{i=1}^{N_{t}} \mathrm{E}\left(\exp \left(-\lambda Y_{i}\right)\right)=\prod_{i=1}^{N_{t}} \mathrm{e}^{-\lambda}=\exp \left(-\lambda N_{t}\right)=X_{t} .
$$

But, if $N_{t+1} \neq N_{t+1}^{\prime}$ then $K=N_{t+1}<N_{t+1}^{\prime}$. Therefore,

$$
0 \leq \mathrm{E}\left(\exp \left(-\lambda N_{t+1}\right)-\exp \left(-\lambda N_{t+1}^{\prime}\right) \mid N_{t}\right) \leq \mathrm{e}^{-\lambda K},
$$

so $\mathrm{E}\left(X_{t+1} \mid N_{t}\right) \leq X_{t}+\mathrm{e}^{-\lambda K}$, and, thus, $\mathrm{E}\left(X_{t+1}\right) \leq \mathrm{E}\left(X_{t}\right)+\mathrm{e}^{-\lambda K}$. But $\mathrm{E}\left(X_{t}\right) \geq \mathrm{P}\left(N_{t}=0\right)$ and $\mathrm{E}\left(X_{0}\right)=\exp \left(-\lambda N_{0}\right)=\mathrm{e}^{-\lambda}$. Hence,

$$
\mathrm{P}\left(N_{T}=0\right) \leq \mathrm{E}\left(X_{T}\right) \leq \mathrm{e}^{-\lambda}+T \mathrm{e}^{-\lambda K} \leq\left(1+\frac{\lambda}{2}\right) \mathrm{e}^{-\lambda} \leq \mathrm{e}^{-\lambda / 2}
$$

and $\mathrm{P}\left(N_{T}>0\right) \geq 1-\mathrm{e}^{-\lambda / 2}$, as required. 
We now consider a simplified version of our percolation process in which each step is independent of all previous steps. We define a branching process of nodes. Each node is assigned a region $A_{v}$ independently of all previous nodes according to the distribution $\mathscr{D}$, and then branches into a number of new nodes, where the number of child nodes is Poisson distributed with mean $\left|A_{v}\right|$. As a consequence, the number of child nodes has overall distribution $\mathscr{D}_{0}$. Also, for each child node $u$ of $v$, we choose $\delta_{u}$ uniformly from the set $A_{v}$ independently of all other $\delta_{u}$ s. Unconditioned on $A_{v}, \delta_{u}$ then has probability distribution $\mathscr{D}_{c}$. Fix a position $z_{0}$ in $\mathbb{R}^{d}$ for the root node, and define the position $z_{v}$ of a node $v$ to be $z_{0}+\sum_{u} \delta_{u}$, where the sum runs over all predecessors $u$ of $v$ back to the root node. Let $\mathcal{T}$ be the random graph with vertices $z_{v}$ and edges $z_{v} z_{u}$ for all child nodes $u$ of $v$. Set $\mathcal{T}^{t}$ to be the set of nodes that are $t$ steps from the root node in the graph $\mathcal{T}$. The process $\mathcal{T}$ approximates the percolation process $g$, but it differs in that the distribution of points (child nodes) in $A\left(z_{v}\right)=A_{v}+z_{v}$ is independent of the process up to that point, whereas in $g$ the points in $A\left(z_{v}\right)$ will depend on points in previously encountered regions $A\left(z_{u}\right)$ where they intersect. Effectively, the difference between $\mathcal{T}$ and $g$ is that when we encounter a node $z_{u}$ in $\mathcal{T}$ we 'regenerate' the Poisson process in the whole of $A\left(z_{u}\right)$, whereas in $g$ we only generate a Poisson process in the region of $A\left(z_{u}\right)$ that we have not already seen. To simplify the notation, we shall generally identify a node $u$ with its position $z_{u} \in \mathbb{R}^{d}$, so, for example, we shall denote $A\left(z_{u}\right)$ by $A(u)$.

In $\mathcal{T}$, any path from the root gives rise to a random walk in $\mathbb{R}^{d}$. We shall analyse this walk by comparison with a Brownian motion. Recall that a Brownian motion (with drift) is a continuous-time stochastic process $B_{t} \in \mathbb{R}$ such that, for all $t_{1}>t_{2}, B_{t_{1}}-B_{t_{2}}$ is given by a normal distribution with mean $\beta\left(t_{2}-t_{1}\right)$ and variance $\gamma\left(t_{2}-t_{1}\right)$, and is independent of $B_{t}$ for $t<t_{1}$. We call $\beta$ the drift and $\gamma$ the unit time variance of $B_{t}$. We start with a well-known result.

Lemma 6. If $B_{t}$ is a Brownian motion with drift $\beta$ and unit time variance $\gamma$, then the probability of $B_{t}$ hitting 1 before hitting 0 starting at $x \in[0,1]$ is given by

$$
f(x)=\frac{1-\mathrm{e}^{-2 x \beta / \gamma}}{1-\mathrm{e}^{-2 \beta / \gamma}} \quad(\beta \neq 0) \quad \text { or } \quad f(x)=x \quad(\beta=0) .
$$

Proof. Let $f(x)$ be the solution of the equation

$$
\frac{\gamma}{2} \frac{\mathrm{d}^{2} f}{\mathrm{~d} x^{2}}+\beta \frac{\mathrm{d} f}{\mathrm{~d} x}=0, \quad f(0)=0, \quad f(1)=1 .
$$

Then $\mathrm{E}\left(f\left(B_{t+\delta t}\right)-f\left(B_{t}\right) \mid B_{t}=x\right)=\mathrm{E}(f(x+Z)-f(x))$, where $Z$ is normal with mean $\beta \delta t$ and variance $\gamma \delta$ t. By solving the above differential equation we see that $\left|f^{\prime \prime \prime}(x)\right|=O\left(C^{|x|}\right)$ for some $C$. Thus, $f(x+Z)-f(x)=Z f^{\prime}(x)+\frac{1}{2} Z^{2} f^{\prime \prime}(x)+O\left(Z^{3} C^{|Z|}\right)$, and

$$
\mathrm{E}(f(x+Z)-f(x))=\beta \delta t f^{\prime}(x)+\frac{1}{2}\left(\gamma \delta t+\beta^{2} \delta t^{2}\right) f^{\prime \prime}(x)+O\left(\delta t^{3 / 2}\right)=o(\delta t) .
$$

Taking $\delta t \rightarrow 0$ and summing between $t=t_{1}$ and $t=t_{2}$, we see that $\mathrm{E}\left(f\left(B_{t_{2}}\right) \mid f\left(B_{t_{1}}\right)\right)=$ $f\left(B_{t_{1}}\right)$ for all $t_{2}>t_{1}$, i.e. $f\left(B_{t}\right)$ is a martingale. Since $f$ is bounded on $[0,1]$ and $B_{t}$ is almost surely continuous, $f(x)=\mathrm{E}\left(f\left(B_{0}\right)\right)=\mathrm{E}\left(f\left(B_{T}\right)\right)$, where $T$ is the stopping time inf $\left\{t: B_{t}=0\right.$ or 1 \}. But $f(0)=0$ and $f(1)=1$, so this is just the probability that $B_{t}$ hits 1 before hitting 0 . Solving the above differential equation gives the result.

Lemma 7. There exist absolute constants $c_{1}, c_{2}, c_{3}>0$ with the following property. Assume that we are given a random walk $Z_{i}$ in $\mathbb{R}^{2}$ with independent and identically distributed steps 
$Y_{i}=Z_{i+1}-Z_{i}$. Assume that the mean step size $\mathrm{E}\left(Y_{i}\right)$ is either 0 or in the direction $(1,1)$ and that there is a constant $r>0$ such that, for any unit vector $u, r^{2} \operatorname{var}\left(Y_{i} \cdot u\right)+\left(\mathrm{E}\left(Y_{i} \cdot u\right)\right)^{2}=r^{4}$. Assume further that the maximum step size is at most $c_{1}$ and that the random walk starts at $Z_{0} \in[-2,2] \times[-2,2]$. Then the probability of the random walk entering $[5,7] \times[-1,1]$ before getting within $c_{1}$ of the boundary of the rectangle $[-3,9] \times[-3,3]$ and before time $c_{2} / r^{2}$ is at least $c_{3}$.

Proof. Introduce the coordinates $x_{1}=(x+y) / \sqrt{2}$ and $x_{2}=(y-x) / \sqrt{2}$, so the $\left(x_{1}, x_{2}\right)$ coordinates are obtained from the $(x, y)$ coordinates by a rotation of 45 degrees. Then $\mathrm{E}\left(Y_{i}\right)$ lies on the $x_{1}$-axis. Assume that $\left\|\mathrm{E}\left(Y_{i}\right)\right\|=\alpha r^{2}$, so the variance of the step size is $\left(1-\alpha^{2}\right) r^{2}$ in the $x_{1}$-direction and $r^{2}$ in the $x_{2}$-direction. Note that the maximum step size must be at least $r$, so $r \leq c_{1}$. Consider a two-dimensional Brownian motion with independent $x_{1}$ and $x_{2}$ coordinates, drift $\alpha$ in the $x_{1}$-direction, unit time variance $1-\alpha^{2}$ in the $x_{1}$-direction, and unit time variance 1 in the $x_{2}$-direction. Let $f_{\alpha}(z)$ be the probability of this Brownian motion starting at $z \in \mathbb{R}^{2}$ hitting $D=[5.1,6.9] \times[-0.9,0.9]$ before leaving $C=[-3,9] \times[-3,3]$ (see Figure 2). Then arguing as in Lemma 6 we can show that $f_{\alpha}(z)$ satisfies the following partial differential equation:

$$
\frac{1}{2} \frac{\partial^{2} f_{\alpha}}{\partial x_{2}^{2}}+\frac{1}{2}\left(1-\alpha^{2}\right) \frac{\partial^{2} f_{\alpha}}{\partial x_{1}^{2}}+\alpha \frac{\partial f_{\alpha}}{\partial x_{1}}=0 .
$$

Now, for $\alpha \in[0,1], f_{\alpha}(z)$ is a smooth function in the interior of $C \backslash D$ which is 1 on $\partial D$, 0 on $\partial C$, and $0 \leq f_{\alpha}(z) \leq 1$ on $C \backslash D$. Note that $f_{\alpha}(z)>0$ in $[-2,2] \times[-2,2]$ for all $\alpha$; indeed $f_{\alpha}(z)>0$ for all $z$ in the interior of $C \backslash D$ except in the region $x_{1} \geq 7.8 / \sqrt{2}$ when $\alpha=1$. Choose $c_{3}>0$ so that

$$
3 c_{3} \leq \inf \left\{f_{\alpha}(x, y):(x, y) \in[-2,2] \times[-2,2], \alpha \in[0,1]\right\} .
$$
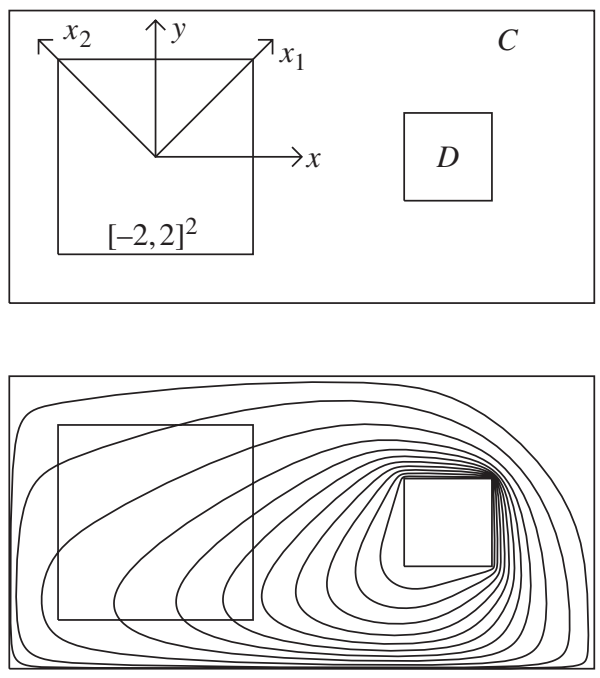

$\alpha=0.8$

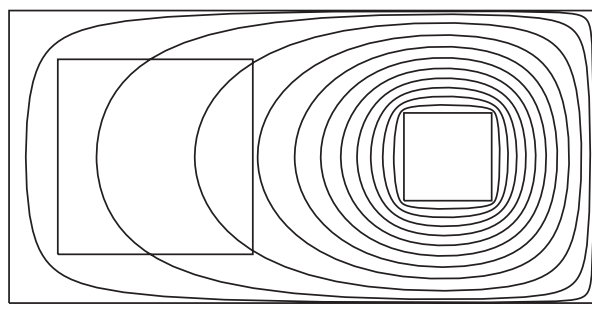

$\alpha=0$

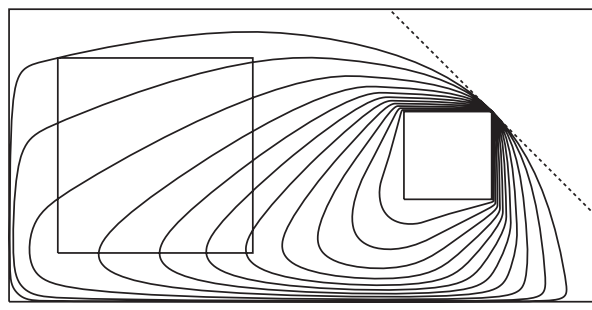

$\alpha=1$

Figure 2: Function $f_{\alpha}$ in $C$. Curves are the contours $f_{\alpha}=0.005,0.03,0.1,0.2, \ldots, 0.9$. Values of $f_{\alpha}$ are strictly positive in $C$ except when $\alpha=1$ and $x_{1} \geq 7.8 / \sqrt{2}$. 
We shall assume that $c_{3}<0.1$. As $z$ approaches $\partial C, f_{\alpha}(z)$ tends to 0 , uniformly in both $z$ and $\alpha$. To see this, note that, for $y \in[-3,-2], f_{\alpha}(x, y)$ is at most the probability of the Brownian motion hitting $y=-2$ before $y=-3$, since if it hits $y=-3$ then it has definitely hit $\partial C$, whereas to hit $\partial D$ it must have hit $y=-2$ first. If we project the Brownian motion onto the $y$-coordinate, we obtain a Brownian motion with drift $\alpha / \sqrt{2}$ and unit time variance $1-\alpha^{2} / 2$, so by Lemma 6 we have

$$
f_{\alpha} \leq \frac{1-\exp \left(-\varepsilon \alpha \sqrt{2} /\left(2-\alpha^{2}\right)\right)}{1-\exp \left(-\alpha \sqrt{2} /\left(2-\alpha^{2}\right)\right)}
$$

when we are within $\varepsilon$ of the $y=-3$ side of $\partial C$. This expression is maximised when $\alpha=1$, so we obtain $f_{\alpha} \leq\left(1-\mathrm{e}^{-\varepsilon \sqrt{2}}\right) /\left(1-\mathrm{e}^{-\sqrt{2}}\right) \leq 1.87 \varepsilon$. A similar argument applies starting with $y \in[2,3]$, except in this case the drift helps us and the maximum occurs at $\alpha=0$ with $f_{\alpha} \leq \varepsilon$ when we are within $\varepsilon$ of $y=3$. Projecting onto the $x$-coordinate deals with the remaining two sides of $\partial C$ and gives smaller bounds. Thus, if $c_{4}>0$ is sufficiently small $\left(c_{4} \leq c_{3} / 1.87\right)$, we can assume that $\left|f_{\alpha}(x)\right|<c_{3}$ when $d(x, \partial C)<c_{4}$. We shall assume that $c_{4}<0.1$. Since all derivatives of $f_{\alpha}(x)$ exist and are continuous on the interior of $C \backslash D$, we can assume that the second and third directional derivatives $D_{u}^{2} f_{\alpha}(x)$ and $D_{u}^{3} f_{\alpha}(x)$ are bounded by a constant $M$, uniformly in $u, x$, and $\alpha$, provided that $x$ is at a distance at least $c_{4} / 2$ from the boundary of $C \backslash D$. Provided that $Z_{i}$ is further than $c_{4}$ from $\partial C \cup \partial D$, we can estimate $\mathrm{E}\left(f_{\alpha}\left(Z_{i+1}\right) \mid Z_{i}\right)$ by approximating $f(x)$ near $x=Z_{i}$. Indeed, if $c_{1}<c_{4} / 2$ then $Z_{i+1}$ must be at least $c_{4} / 2$ from $\partial C \cup \partial D$ and so

$$
f_{\alpha}\left(Z_{i+1}\right)-f_{\alpha}\left(Z_{i}\right)=\sum_{j=1}^{2} u_{j} \frac{\partial f_{\alpha}}{\partial x_{j}}+\frac{1}{2} \sum_{j, k=1}^{2} u_{j} u_{k} \frac{\partial^{2} f_{\alpha}}{\partial x_{j} \partial x_{k}}+\theta\left(Y_{i}\right)\left\|Y_{i}\right\|^{3}
$$

where $Y_{i}=\left(u_{1}, u_{2}\right)$ and $\left|\theta\left(Y_{i}\right)\right| \leq M / 6$. However, $\mathrm{E}\left(u_{1}\right)=\alpha r^{2}, \mathrm{E}\left(u_{2}\right)=\mathrm{E}\left(u_{1} u_{2}\right)=0$, $\mathrm{E}\left(u_{1}^{2}\right)=\left(1-\alpha^{2}\right) r^{2}+\alpha^{2} r^{4}$, and $\mathrm{E}\left(u_{2}^{2}\right)=r^{2}$, so

$$
\begin{aligned}
& \mathrm{E}\left(f_{\alpha}\left(Z_{i+1}\right)-f_{\alpha}\left(Z_{i}\right) \mid Z_{i}\right) \\
& \quad=\alpha r^{2} \frac{\partial f_{\alpha}}{\partial x_{2}}+\frac{1}{2}\left(\left(1-\alpha^{2}\right) r^{2}+\alpha^{2} r^{4}\right) \frac{\partial^{2} f_{\alpha}}{\partial x_{2}^{2}}+\frac{1}{2} r^{2} \frac{\partial^{2} f_{\alpha}}{\partial x_{1}^{2}}+\mathrm{E}\left(\theta\left(Y_{i}\right)\left\|Y_{i}\right\|^{3}\right) \\
& \quad=\frac{1}{2} \alpha^{2} r^{4} \frac{\partial^{2} f_{\alpha}}{\partial x_{2}^{2}}+\mathrm{E}\left(\theta\left(Y_{i}\right)\left\|Y_{i}\right\|^{3}\right) .
\end{aligned}
$$

Since $r \leq c_{1}<1$ and $\alpha \leq 1$, we obtain

$$
\left|\mathrm{E}\left(f_{\alpha}\left(Z_{i+1}\right)-f_{\alpha}\left(Z_{i}\right) \mid Z_{i}\right)\right| \leq \frac{1}{2} \alpha^{2} r^{4} M+\frac{1}{6} M c_{1} r^{2} \leq M c_{1} r^{2}
$$

Run the random walk until the stopping time $T=\min \left\{i: d\left(Z_{i}, \partial C \cup \partial D\right)<c_{4}\right\}$. We shall now show that $T$ is unlikely to be very large. Writing $\left(Z_{t}\right)_{2}$ for the $x_{2}$-coordinate of $Z_{t}$ we have $\mathrm{E}\left(\left(Z_{t+1}\right)_{2}^{2} \mid Z_{t}\right)=\left(Z_{t}\right)_{2}^{2}+r^{2}$, so if we set $g(t)=\mathrm{E}\left(\left(Z_{\min (t, T)}\right)_{2}^{2}\right)$ then $g(t+1)=$ $g(t)+r^{2} \mathrm{P}(T>t)$. Since $\left(Z_{\min (t, T)}\right)_{2}^{2}$ is bounded above by $\sup _{C} x_{2}^{2}=(12 / \sqrt{2})^{2}=72$ and 
$\mathrm{P}(T>t)$ is monotonically decreasing in $t$, we have $t r^{2} \mathrm{P}(T>t) \leq g(t+1)-g(1) \leq 72$ for all $t$. Hence, if $c_{2} \geq 72 / c_{3}, \mathrm{P}\left(T>c_{2} / r^{2}\right) \leq c_{3}$. Since $f_{\alpha}(z)$ is bounded between 0 and 1 ,

$$
\begin{aligned}
\mathrm{E}\left(f_{\alpha}\left(Z_{T}\right)\right) & \geq \mathrm{P}\left(T \leq \frac{c_{2}}{r^{2}}\right) \mathrm{E}\left(f_{\alpha}\left(Z_{\min \left\{T, c_{2} / r^{2}\right\}}\right)\right) \\
& \geq\left(1-c_{3}\right)\left(f_{\alpha}\left(Z_{0}\right)-M c_{1} r^{2} \frac{c_{2}}{r^{2}}\right) \\
& \geq 0.9\left(3 c_{3}-M c_{1} c_{2}\right),
\end{aligned}
$$

which is at least $2 c_{3}$ for sufficiently small $c_{1}\left(c_{1}<0.7 c_{3} / M\right.$ is enough). Hence, if $p=$ $\mathrm{P}\left(d\left(Z_{T}, \partial D\right)<c_{4}\right)$ then $(1)(p)+\left(c_{3}\right)(1-p) \geq \mathrm{E}\left(f_{\alpha}\left(Z_{T}\right)\right) \geq 2 c_{3}$, so $p \geq c_{3}$. Therefore, with probability at least $c_{3}$, the random walk gets closer than $c_{4}$ to $D$ (and, hence, enters $[5,7] \times[-1,1])$ before time $c_{2} / r^{2}$ and before getting within $c_{4}>c_{1}$ of the boundary of the rectangle $[-3,9] \times[-3,3]$.

The numerical values of $c_{1}, c_{2}$, and $c_{3}$ given by this proof are not very good. Indeed, simulations suggest that the bound on $3 c_{3}$ given by (4) is just over 0.005 , so we can take $c_{3}=\frac{1}{600}, c_{4}=\frac{1}{1200}$, and $c_{2}=43200$. We can show that, for this $c_{4}$, we can set $M=10^{23}$ (the worst case is near the $(6.9,0.9)$ corner of $D$ when $\alpha=1)$, and then we can take $c_{1}=10^{-31}$.

Lemma 8. Assume that $c_{1}, c_{2}$, and $c_{3}$ are as in Lemma 7. Consider the branching process $\mathcal{T}$ defined above where $z_{0}$ lies in $C_{x, 2}$. Run $\mathcal{T}$ for time $T=\left\lfloor c_{2} R^{2} / r_{s}^{2}\right\rfloor$, except that at each step (if necessary) we remove nodes (randomly, independent of their positions) so there are at most $K$ nodes left from $\mathcal{T}^{t}$. Assume that $R \geq r_{1} / c_{1}$, and let $\&$ be the event that, for a node $w$ uniformly randomly chosen from $\mathcal{T}^{T}$, some ancestor $v$ of $w$ lies in the region $C_{y, 1}$ and all ancestors of $v$ lie in $C_{x y}^{o}$. Then $\mathrm{P}\left(\& \mid \mathcal{T}^{T} \neq \varnothing\right) \geq c_{3}$.

Proof. Conditioning on $\mathcal{T}^{T} \neq \varnothing$, pick a node $w$ uniformly at random from $\mathcal{T}^{T}$. Then, for any $t<T$, the unique ancestor of $w$ in $\mathcal{T}^{t}$ is equally likely to be any element of $\mathcal{T}^{t}$. (The number of descendants of each $u \in \mathcal{T}^{t}$ is independent of the process up to $u$.) Hence, the locations of the nodes on the path from the root node to $w$ form a random walk with steps taken from the distribution $\mathscr{D}_{c}$. If we project onto the first two coordinates, translate so that the centre of $C_{x}$ is at the origin, and scale by a factor of $1 / R$, this random walk will start in $[-2,2] \times[-2,2]$, have maximum step size $r_{1} / R \leq c_{1}$, and, for any unit vector $u, r^{2} \operatorname{var}(Y \cdot u)+(\mathrm{E}(Y \cdot u))^{2}=r^{4}$, where $r=r_{s} / R$. The drift (if any) is in the $(1,1)$ direction. By Lemma 7 , with probability at least $c_{3}$, the walk enters $[5,7] \times[-1,1]$ before getting within $c_{1}$ of the boundary of $[-3,9] \times[-3,3]$ and before time $c_{2}\left(R / r_{s}\right)^{2}$. Thus, with probability at least $c_{3}$, the original walk enters $C_{y, 1}$ before getting within $c_{1} R \geq r_{1}$ of the boundary of $C_{x} \cup C_{y}$ and before time $T$.

Theorem 3. There exists an absolute constant $c>0$ such that if $\delta<c r_{s}^{9} r_{1}^{-9} \eta^{16} \sigma^{-32}$ then we can choose $N, n$, and $R=C r_{1}$ such that the probability of a bond being open with respect to any $P$ and $Q$ satisfying assumptions (A1)-(A3), conditioned on the choices of $A(z), z \in Q$, and the Poisson process outside of $C_{x y}^{o} \backslash \bigcup_{z \in Q} A(z)$, is at least 0.9 .

Proof. The strategy of the proof is to find some path from $x_{i} \in P$ to some point $x_{i}^{\prime \prime}$ in $C_{y, 1}$ in the $\mathcal{T}$ process, and by coupling, show that, with reasonable probability, a corresponding path will exist in the $g$ process. We shall then couple the percolation for $R / r_{1}$ further steps to obtain at least $n$ points $x_{j}^{\prime}$. These will lie in $C_{y, 2}$, as we cannot travel more than a distance $R$ in $R / r_{1}$ steps. Since the probability of success is fairly small, we apply this process to each $x_{i} \in P$ and show that it is very likely that we will succeed for at least one $x_{i}$. 
Run $n$ independent truncated branching processes for $T$ steps starting at the points $x_{1}, \ldots, x_{n}$ as in Lemma 8, where

$$
\begin{gathered}
T=\left\lfloor\frac{c_{2} R^{2}}{r_{s}^{2}}\right\rfloor, \\
K=\left\lceil\frac{1}{\lambda} \log \frac{2 T}{\lambda}+1\right\rceil .
\end{gathered}
$$

Let $\mathcal{T}=\bigcup_{i=1}^{n} \mathcal{T}_{i}$ be the union of these processes, so that $\mathcal{T}$ is a union of $n$ trees, with the $i$ th tree $\mathcal{T}_{i}$ starting at $x_{i}$. We shall couple $\mathcal{T}$ with $\mathcal{g}_{\text {one }}$ onel at a time, and within each level $\mathcal{T}^{t}$, sequentially run through all nodes in the previous level, and couple all the children of this node, one at a time (i.e. after coupling one node, we continue with each of its siblings before processing any other node). We shall declare some nodes $u$ to be good. For these nodes, the positions and choice of $A_{u}$ will have been successfully coupled so that they are equal to the corresponding values in the $g$ process. However, we shall also require them to avoid certain sets $B(z)$, and for their sets $B(u)$ to avoid certain other nodes. We shall not attempt to couple the descendants of a node $u$ or the process inside $A(u)$ unless $u$ is good. (Technically, we couple the process independently with no requirement that the existence of $v$, position of $v$, or region $A(v)$ defined in the $\mathcal{T}$ process matches with anything in the $g$ process when $v$ is a descendant of a bad node $u$.)

The set $Q(u)$ will be the points of $Q$ together with the set of all good nodes that have had all their children coupled before $u$. Before coupling $u$ we will have fixed the Poisson process in $\bigcup_{z \in Q(u)} A(z)$ and outside $C_{x y}^{o}$. Let $P(u)$ be the set of good nodes found that are not in $Q(u)$. These are nodes which have uncoupled children, and in particular include $p_{u}$, the parent of $u$. For these nodes (other than $p_{u}$ ), we have not yet fixed the Poisson process in the $A(z)$ (see Figure 3).

We shall ensure that we do not consider more than $N$ new points of $g$ in $C_{x y}^{o}$, where

$$
N=n\left(K T+\left\lfloor\frac{R}{r_{1}}\right\rfloor\right) .
$$

Hence, in particular, $|Q(u) \cup P(u)| \leq N+|Q| \leq 4 N$ for all $u$.

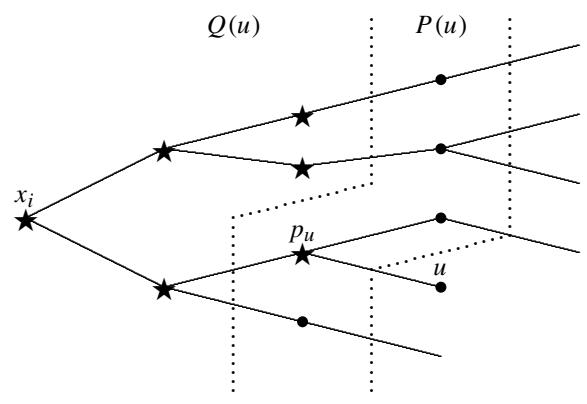

Figure 3: The situation just before processing node $u$ of $\mathcal{T}$. All nodes $v \in P(u) \cup Q(u)$ have been coupled to points $x_{v}$ in $g$, and the choice of $A_{v}$ has been matched to that of $A\left(x_{v}\right)$. For nodes marked with a ' $\star$ ', we have also coupled the Poisson process in $A\left(x_{v}\right)$ where it does not overlap previously fixed regions. 
Step 1. We start by coupling the choices of $A_{x_{i}}$ in $\mathcal{T}$ to match those in $g_{\text {for }}$ forch $x_{i} \in P$. Recall that $A_{x_{i}}$ determines $B\left(x_{i}\right)$. Define $\varepsilon_{i}$ to be the event that $x_{j} \in B\left(x_{i}\right)$ for some $x_{j} \neq x_{i}$. By assumption (A3), the probability of $\varepsilon_{i}$ is bounded above is

$$
p_{1}=n \sqrt[4]{\delta}
$$

If $\varepsilon_{i}$ occurs, we declare $x_{i}$ to be bad, and we do not couple the process in $A\left(x_{i}\right)$, or any descendant of $x_{i}$. Otherwise, $x_{i}$ is good. After step 1, but before processing the first child $u$ of the first $x_{i}$, we have $Q(u)=Q$, and $P(u)=P_{\mathrm{g}}$ is the subset of good nodes in $P$.

Step 2. We process each node of $\mathcal{T}$ in turn as described above, but before coupling any children of $p_{u}$ we check if $p_{u} \in C_{y, 1}$. In this case we do not couple any children of $p_{u}$, and $p_{u}$ will remain in $P(v)$ for all subsequent nodes $v$.

Step 3. Couple the Poisson process in $A\left(p_{u}\right)$ in $\mathcal{T}$ with the same region in $g$ so that they are the same where $A\left(p_{u}\right)$ does not overlap previously coupled regions $A(z)$, or lies in one of the areas we have conditioned on (i.e. they should agree in $C_{x y}^{o} \backslash \bigcup_{z \in Q(u)} A(z)$ ).

Step 4. For each child $u$ of $p_{u}$, we couple the position of $u$ in $\mathbb{R}^{d}$ to the position of a point in $g$ provided that

$$
u \in C_{x y}^{o}, \quad u \notin A(z) \quad \text { for all } z \in Q(u), \quad u \notin B(z) \quad \text { for all } z \in Q(u) \cup P(u) .
$$

Otherwise the node $u$ will be bad and we shall not couple the process in the region $A(u)$, or any descendant of $u$. Note that (by step 3 ) the first two conditions are essential if we are to couple the $\mathcal{T}$ and $g$ processes so that the locations of the nodes agree with the points in $g$. The last condition $(u \notin B(z))$ will be used to ensure that the children of $u$ are unlikely to lie in some previously coupled set $A(z)$.

Step 5. Couple $A_{u}$ with the corresponding choice in $g$. This now defines $B(u)$. We require that

$$
z \notin B(u) \text { for all } z \in P(u) \text {. }
$$

Otherwise, we again call $u$ bad and ignore it and all its descendants. The reason is that if $z \in B(u)$ then coupling the descendants of $z$ would be prejudiced.

Repeat steps 4 and 5 for each child of $p_{u}$ before continuing with the next parent node (with step 2). Note that, for $x_{i} \in P_{\mathrm{g}}$, we do not need to (and cannot) insist that $x_{i} \notin A(z)$ for all $z \in Q$. However, by step 1 and assumption (A2), $x_{i} \notin B(z)$ for all $z \in Q \cup P_{\mathrm{g}}$.

Assume that $\mathcal{T}_{i}^{T} \neq \varnothing$, and pick a node uniformly at random from $\mathcal{T}_{i}{ }^{T}$. We wish to estimate the probability that all nodes on the path from $x_{i}$ to this node are good. Consider a node $u$ on this path and assume that all its predecessors are good. The parent $p_{u}$ does not lie in $B(z)$ for any $z \in Q(u)$, since $Q(u) \subseteq Q\left(p_{u}\right) \cup P\left(p_{u}\right)$ and $p_{u}$ is good (or since $Q(u) \subseteq Q \cup P_{\mathrm{g}}$ and $p_{u}$ is good in the case where $p_{u}=x_{i} \in P$ ). However, $u-p_{u}$ is distributed according to $\mathscr{D}_{c}$, so by Lemma 2 and the definition of $\mathscr{D}_{c}$, the probability that $u \in A(z)$ for some $z \in Q(u)$ is bounded above by $|Q(u)| \sqrt{\delta} \leq 4 N \sqrt{\delta}$.

By Lemma 2, the probability that $u \in B(z)$ for some $z \in Q(u) \cup P(u)$ is also at most $4 N \sqrt{\delta}$. Note that if $z$ is a sibling of $u$ then we must assume that $A\left(p_{u}\right)$ is fixed, so we need the full strength of Lemma 2 , that is, that $\left|\left(A+p_{u}\right) \cap B(z)\right| \leq \sqrt{\delta}|A|$ for almost all $A$, not just averaged over $A$.

Finally, the probability that $z \in B(u)$ for some $z \in P(u)$ is at most $4 N \sqrt{\delta}$, since, even conditioned on $A_{u}$ and $A_{p_{u}}$, the probability that a fixed $z$ lies in $B(u)$ is the same as the probability that $z-u$ lies in $B_{u}=B(u)-u$, and $z-u$ is uniformly distributed in $\left(z-p_{u}\right)-A_{p_{u}}$. By Lemma 2, the probability that $z \in B(u)$ is then at most $\sqrt{\delta}$ for each $z$. 
Ignoring the possibility that we have left $C_{x y}^{o}$ or entered $C_{y, 1}$, the probability that $\varepsilon_{i}$ did not occur, but we failed to couple the path in $\mathcal{T}_{i}$ with $g$, is at most $12 N T \sqrt{\delta}$. We shall require that the parameters $n$ and $R$ are chosen below so that

$$
12 N T \sqrt{\delta} \leq \frac{c_{3}}{2},
$$

where $c_{3}$ is the constant given in Lemma 7.

Now, conditioning on $\mathcal{T}_{i}^{T} \neq \varnothing$, the above path in $\mathcal{T}_{i}$ hits $C_{y, 1}$ before leaving $C_{x y}^{o}$ with probability at least $c_{3}$. Hence, with probability at least $c_{3}-c_{3} / 2=c_{3} / 2$, either $\mathcal{E}_{i}$ occurred or the coupling above stopped at a good node $x_{i}^{\prime \prime} \in C_{y, 1}$. Now put all the other coupled nodes of $\mathcal{T}_{i}$ into $Q(u)$ and run another $\mathcal{T}$ process starting at the points $x_{i}^{\prime \prime}, i=1, \ldots, n$, that are good and lie in $C_{y, 1}$. We run this second $\mathcal{T}$ process for another $\left\lfloor R / r_{1}\right\rfloor$ steps. We couple this $\mathcal{T}$ process with $g$ as above. Of course, this time we do not stop coupling if we hit $C_{y, 1}$, and we cannot leave $C_{x y}^{o}$ (or even $C_{y, 2}$ ) in $\left\lfloor R / r_{1}\right\rfloor$ steps. One minor difference is that in each level we do not couple the $A(u) \mathrm{s}$, and, hence, we do not insist on $z \notin B(u)$ for $z$ and $u$ in the current level, until all nodes from that level are processed. If there are at least $n$ nodes left in this level, we stop and set $P^{\prime}$ equal to $n$ of these nodes. Otherwise, we apply step 5 to all the good nodes of this level (checking both the $u \notin B(z)$ and $z \notin B(u)$ conditions of step 4 and step 5) before starting the next level. The reason for this complication is that we do not want to couple the choice of $A_{u}$ s for $u \in P^{\prime}$.

Assuming that $x_{i}^{\prime \prime}$ exists, we now estimate the probability that a node $u$ of this new process is bad, conditioned on all its predecessors being good, and on any event involving the existence of its descendants. As above, this probability is at most $12 N \sqrt{\delta}$, and we shall require that

$$
12 N \sqrt{\delta}<\frac{\eta}{2}
$$

Thus, the good nodes stochastically dominate a branching process with one-step mean $1+\eta / 2$, variance at most $\sigma^{2}+(1+\eta)^{2}-(1+\eta / 2)^{2} \leq \sigma^{2}+2 \leq 3 \sigma^{2}$ (as $\left.\eta \leq 1\right)$, and the probability of zero children being at least $p_{0}$. Hence, by Lemma 4 we will have more than $n$ good descendants of $x_{i}^{\prime \prime}$ within time $\left\lfloor R / r_{1}\right\rfloor$ with probability at least $\eta p_{0}^{2} /\left(6 \sigma^{2}\right)$ provided that

$$
n \leq\left(1+\frac{\eta}{2}\right)^{\left\lfloor R / r_{1}\right\rfloor}
$$

If at any level we find $n$ good points (without the condition that $z \notin B(u)$ for $z \in P(u)$ ) then we stop the process and set $P^{\prime}$ to be these $n$ points. Any other good points found will be placed in the set $Q^{\prime}$. The probability that either $\varepsilon_{i}$ occurs or we get $n$ good descendants of $x_{i}$ in $C_{y, 2}$ is now at least

$$
p_{2}=\left(1-\mathrm{e}^{-\lambda / 2}\right)\left(\frac{c_{3}}{2}\right)\left(\frac{\eta p_{0}^{2}}{6 \sigma^{2}}\right),
$$

where $1-\mathrm{e}^{-\lambda / 2}$ bounds the probability that $\mathcal{T}_{i}^{T} \neq \varnothing$ (by Lemma 5$), c_{3} / 2$ bounds the probability that given this either $\mathcal{E}_{i}$ occurs or we obtain a good $x_{i}^{\prime \prime}$, and $\eta p_{0}^{2} /\left(6 \sigma^{2}\right)$ bounds the probability that a good $x_{i}^{\prime \prime}$ has $n$ good descendants at some level.

Since $\mathrm{P}\left(\varepsilon_{i}\right) \leq p_{1}$, we obtain $n$ good descendants of $x_{i}$ with probability at least $p_{2}-p_{1}$, regardless of the success or otherwise at any other $x_{j}$. Hence, we will fail to obtain $n$ good descendants in $C_{y, 2}$ of any $x_{i}$ with probability at most $\left(1-\left(p_{2}-p_{1}\right)\right)^{n} \leq \exp \left(-\left(p_{2}-p_{1}\right) n\right)$. 
This clearly bounds the probability of failing to obtain $n$ good descendants from all $x_{i}$ combined. We shall require that

$$
p_{2} \geq \frac{3.05}{n}
$$

and

$$
n^{2} \sqrt[4]{\delta}<0.05
$$

so that $p_{1}=n \sqrt[4]{\delta} \leq 0.05 / n$ and $\exp \left(-\left(p_{2}-p_{1}\right) n\right) \leq \exp (-3) \leq 0.05$. The number of points considered is at most $n K T$ in the first $\mathcal{T}$ process and at most $n\left\lfloor R / r_{1}\right\rfloor$ in the second $\mathcal{T}$ process. Thus, $\left|Q^{\prime} \cup P^{\prime}\right|=|(Q(u) \cup P(u)) \backslash Q| \leq N$ by (7). Finally, we need to ensure that assumption (A3) holds for the $x_{i}^{\prime}$. Now, for any $A_{u}$, the probability (choosing $u$ uniformly from $A_{v}$ ) that $z \in B(u)$ is at most $\sqrt{\delta}$. Hence, the probability that a random $A_{u}$ generates $\mathrm{P}(z \in B(u)) \geq \sqrt[4]{\delta}$ is at most $\sqrt[4]{\delta}$. Thus, condition (C3) will fail for some pair $\left(x_{i}^{\prime}, x_{j}^{\prime}\right)$ with probability at most $n^{2} \sqrt[4]{\delta} \leq 0.05$. Hence, the bond $x y$ will be open with probability at least $1-0.05-0.05=0.9$. The result now follows provided that we choose the parameters $n$ and $R$ so that (8)-(13) are satisfied. Lemma 3 implies that, for $\eta \leq 1, \lambda=\Theta\left(\eta / \sigma^{2}\right)$ and $p_{0}=\Theta(1)$. Hence, by (11), $p_{2}=\Theta\left(\eta^{2} / \sigma^{4}\right)$ and (12) is satisfied if we take $n=\left\lceil 3.05 / p_{2}\right\rceil=\Theta\left(\sigma^{4} / \eta^{2}\right)$. Equation (10) is then satisfied if we take

$$
R=\Omega\left(\frac{r_{1}}{\eta} \log \left(\frac{\sigma^{4}}{\eta^{2}}\right)\right) ;
$$

however, we shall in fact set

$$
R=\Theta\left(\frac{r_{1} \sigma^{2}}{\eta} \log \left(\frac{\sigma^{2}}{\eta}\right)\right),
$$

which is sufficiently large since $\sigma^{2}>1$. This will ensure that $C=R / r_{1}$ can be taken to be independent of $r_{1}$ and $r_{s}$, and that

$$
\left(\frac{R}{r_{1}}\right)^{2}>n,
$$

which will be used in the proof of Theorem 1. Equations (5)-(7) then imply that

$$
\begin{gathered}
T=\Theta\left(\frac{r_{1}^{2} \sigma^{4}}{r_{s}^{2} \eta^{2}} \log ^{2}\left(\frac{\sigma^{2}}{\eta}\right)\right), \quad K=\Theta\left(\frac{\sigma^{2}}{\eta} \log \left(\frac{r_{1} \sigma^{2}}{r_{s} \eta}\right)\right), \\
\text { and } \quad N=\Theta\left(\frac{r_{1}^{2} \sigma^{10}}{r_{s}^{2} \eta^{5}} \log ^{3}\left(\frac{r_{1} \sigma^{2}}{r_{s} \eta}\right)\right) .
\end{gathered}
$$

Then

$$
N T=\Theta\left(\frac{r_{1}^{4} \sigma^{14}}{r_{s}^{4} \eta^{7}} \log ^{5}\left(\frac{r_{1} \sigma^{2}}{r_{s} \eta}\right)\right),
$$

so that (8), (9), and (13) are then satisfied provided that $\delta=O\left(r_{s}^{9} r_{1}^{-9} \eta^{16} \sigma^{-32}\right)$.

Proof of Theorem 1. Using Theorem 3, we complete the proof. We define the oriented percolation on $\mathbb{Z}^{2}$. Order the bonds in the first quadrant of $\mathbb{Z}^{2}$ by their $l_{1}$ distance from the origin, and, for each distance $k$, order the bonds at distance $k$ from the origin as

$$
(0, k)(0, k+1), \quad(0, k)(1, k), \quad(1, k-1)(1, k), \quad \ldots, \quad(k, 0)(k+1,0) .
$$




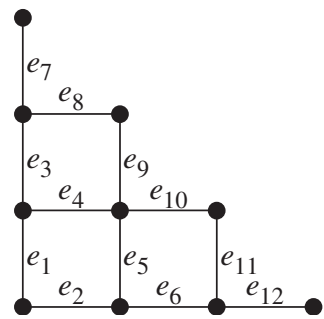

Figure 4: Ordering of the bonds of $\mathbb{Z}^{2}$ in the proof of Theorem 1.

Suppose that the bonds in this order are $\left\{e_{1}, e_{2}, \ldots\right\}$ (see Figure 4). We shall declare some bonds open in such a way that if there is an infinite directed open path in $\mathbb{Z}^{2}$ from $(0,0)$ then (with positive probability) there is an infinite path in $g$. To this end, we shall inductively define, for each bond $e_{i}$, a subset $Q_{i}$ of the Poisson process with $Q_{i-1} \subseteq Q_{i}$, and, for each vertex $x$ joined by an open path to the origin in $\mathbb{Z}^{2}$, a subset $P_{x}$ of the Poisson process inside $C_{x}$.

Initially, set $Q_{0}=\varnothing$, and set $P_{(0,0)}$ to be any subset of $n$ points of the Poisson process that lie in $C_{(0,0), 2}$ and such that each pair of points in $P_{(0,0)}$ is at least $2 r_{1}$ apart. It is easy to check, using (14), that such a set exists with high probability (probability 1 if $d>2$ ).

Now suppose that we have defined the openness of the bonds $e_{j}$ for $j<i$ and the set $Q_{i}$ and all relevant $P_{x}$. We now consider the bond $e_{i}=x y$.

(a) If there is no directed path consisting of open bonds from $(0,0)$ to $x$, set $x y$ to be open and $Q_{i+1}=Q_{i}$.

(b) If there is a directed open path from $(0,0)$ to $x$, declare $x y$ to be open if it is open with respect to $P=P_{x}$ and $Q=Q_{i} \cap\left(C_{x} \cup C_{y}\right)$. If $x y$ is open and $P_{y}$ is not yet defined, set $P_{y}=P^{\prime}$ and $Q_{i+1}=Q_{i} \cup Q^{\prime}$, otherwise set $Q_{i+1}=Q_{i} \cup Q^{\prime} \cup P^{\prime}$.

Condition (a) is a technical condition which clearly does not affect whether or not $(0,0)$ is in an infinite cluster.

By (b), at each step $\left|Q_{i} \cap C_{z}\right| \leq k N$, where $k$ is the number of bonds $e_{j}, j<i$, meeting $z$. Thus, given the ordering of bonds as above, if $e_{i}$ is a vertical bond, $\left|Q_{i} \cap C_{x}\right| \leq 2 N$ and $\left|Q_{i} \cap C_{y}\right| \leq N$, whereas if $e_{i}$ is a horizontal bond, $\left|Q_{i} \cap C_{x}\right| \leq 3 N$ and $\left|Q_{i} \cap C_{y}\right|=0$. Hence, the set $Q$ in (b) always satisfies $|Q| \leq 3 N$.

There are two bonds $e_{i}=x y$ with a given value of $y$, so there are two chances for $P_{y}$ to be defined in (b). Clearly, $P_{y}$ is defined if and only if $y$ is joined to $(0,0)$ by an open path.

If $x$ is joined to $(0,0)$ by an open path $(0,0)=x_{0}, \ldots, x_{k}=x$ then each point in $P_{x_{i+1}}$ is joined by a path in $g$ from a point in $P_{x_{i}}$. Hence, there is a path to any point of $P_{x}$ from one of the $n$ points of $P_{(0,0)}$.

Finally, by Theorem 3, if $\delta<c r_{s}^{9} r_{1}^{-9} \eta^{16} \sigma^{-32}$ then each bond $x y$ is open with probability bounded below by 0.9 even when conditioned on the state of all previous bonds and regions of $\mathbb{R}^{2}$ that they depend on (the $A(z)$ around the points $z \in Q_{i}$ ), except for the other bond starting at $x$. The two bonds starting at $x$ however can be strongly dependent. Define an oriented site percolation on $\mathbb{Z}^{2}$ by declaring $x \in \mathbb{Z}^{2}$ to be open if both oriented bonds from $x$ are open. This process now stochastically dominates an independent oriented site percolation with site probability 0.8 . However, $(0,0)$ is then in an infinite cluster with positive probability (see, for example, [2]). The result now follows. 


\section{References}

[1] Athreya, K. and Ney, P. (1972). Branching Processes. Springer, New York.

[2] Balister, P., Bollobás, B. And Stacey, A. (1994). Improved upper bounds for the critical probability of oriented percolation in two dimensions. Random Structures Algorithms 5, 573-589.

[3] Balister, P., Bollobás, B. and Walters, M. (2004). Continuum percolation with steps in an annulus. Ann. Appl. Prob. 14, 1869-1879.

[4] Balister, P., Bollobás, B. And Walters, M. (2005). Continuum percolation with steps in the square and the disc. Random Structures Algorithms 26, 392-403.

[5] Bollobás, B. (2001). Random Graphs (Camb. Stud. Adv. Math. 73), 2nd edn. Cambridge University Press.

[6] Flaxman, A., Frieze, A. and Upfal, E. (2004). Efficient communication in an ad-hoc network. J. Algorithms 52, 1-7.

[7] Franceschetti, M. et al. (2005). Continuum percolation with unreliable and spread-out connections. J. Statist. Phys. 118, 721-734.

[8] Grimmett, G. (1999). Percolation (Fundamental Principles Math. Sci. 321), 2nd edn. Springer, Berlin.

[9] Meester, R. And Roy, R. (1996). Continuum Percolation (Camb. Tracts Math. 119). Cambridge University Press.

[10] Quintanilla, J., Torquato, S. And Ziff, R. M. (2000). Efficient measurement of the percolation threshold for fully penetrable discs. J. Phys. A 33, L399-L407. 